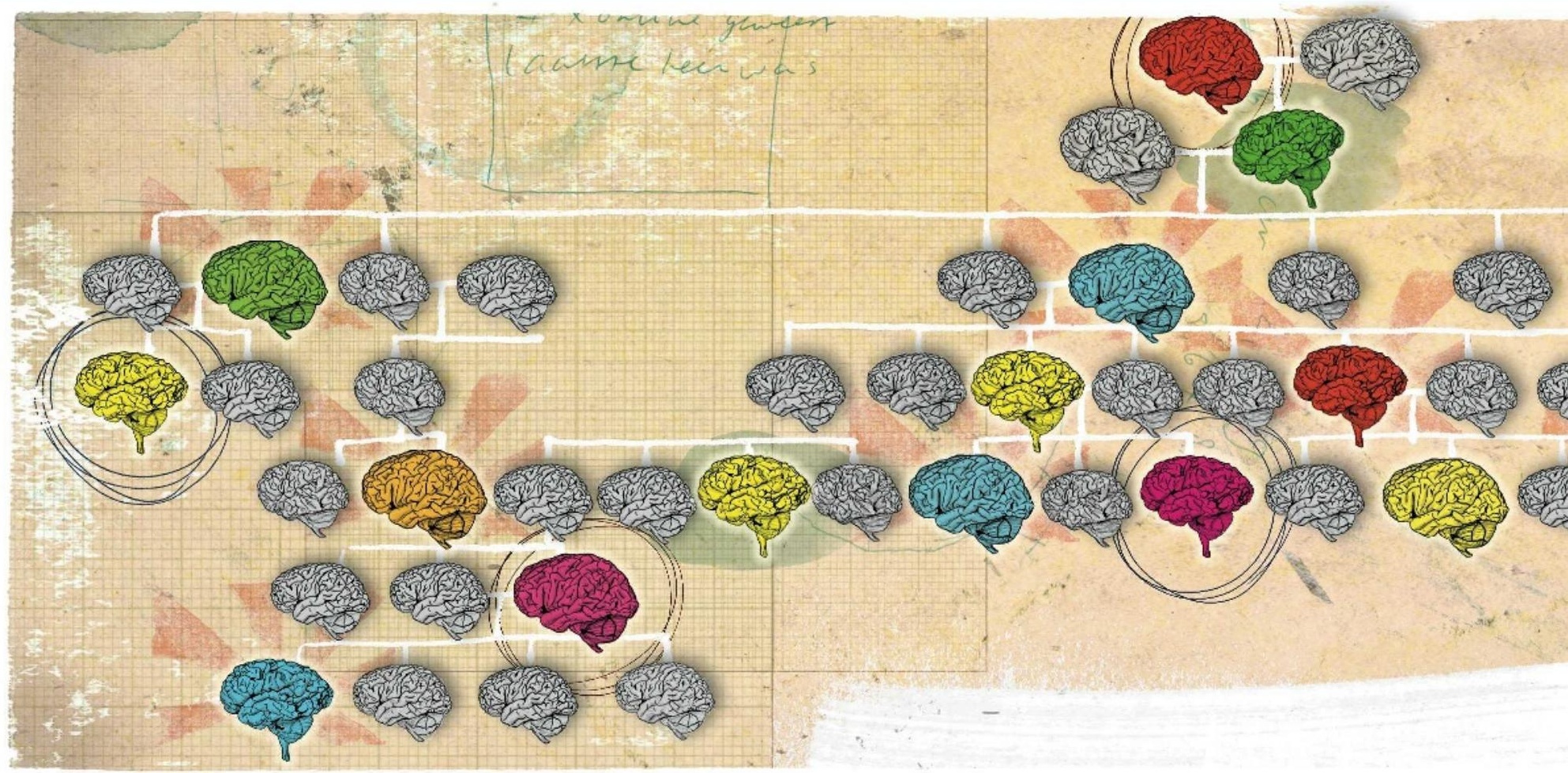

\title{
THE BRAINS OF THE FAMILY
}

\section{Does the difficulty in finding the genes responsible for mental illness reflect the complexity of the genetics or the poor definitions of psychiatric disorders? Alison Abbott reports.}

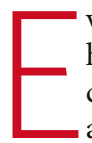
very family has its foibles, but this one has more than most. The first member came to researchers' attention in 1968 as part of a genetic survey of juvenile delinquents who had been admitted to Scottish detention centres. One boy carried a major 'translocation', in which a chunk of chromosome 11 had been switched with part of chromosome 1 .

The translocation and the boy's bad behaviour were more than just coincidence. Years later, when Edinburgh researchers traced the family, they found that the same chromosomal abnormality spanned four generations, with remarkably varied effects. Of those who carried it, five had depression, six had schizophrenia or related disorders, three had adolescent conduct disorder and two had anxiety disorder. One had attempted suicide and died in a mental hospi$\mathrm{tal}^{1}$. Several of those without the translocation had their own problems, including anxiety, minor depressive disorder and alcoholism.

The translocation chops up a gene called, with some justice, disrupted-in-schizophrenia 1 $(D I S C 1)^{2}$. This gene is arguably the strongest contender for involvement in any psychiatric disease, and a flurry of activity has shown that it codes for a hub protein involved in multiple biochemical pathways in the nervous system. But the association and its Scottish heritage are also a textbook example of the complexity of psychiatric genetic studies. DISC1 has not shown up in many other screens for schizophrenia genes, even though it has occasionally been identified in genetic studies of other illnesses, including bipolar disorder and autism.

Researchers are convinced that variations in DISC1 and many other genes can scramble the intricate and as yet inscrutable processes by which brain circuits develop and function, and that this scrambling leaves some people at greater risk of psychiatric problems than others. Even if one or more risk genes are present, external stress or some other event may decide whether and what symptoms are triggered.

Finding genes involved in psychiatric conditions is proving to be particularly intractable because it is still unclear whether the various diagnoses are actually separate diseases with distinct underlying genetics or whether, as the DISC1 story suggests, they will dissolve under the genetic spotlight into one biological continuum. Indeed, some researchers suggest that it would be better to abandon conventional clinical definitions and focus instead on 'intermediate phenotypes', quantifiable characteristics such as brain structure, wiring and function that are midway between the risk genes involved and the psychopathology displayed.
In the past two years, researchers have pulled out a host of genes involved in other multifactorial diseases, such as diabetes and obesity, by use of genome-wide association studies. These use powerful new genomic tools to scan for variations in the DNA sequence called single nucleotide polymorphisms (SNPs) that tend to occur in individuals $\frac{\bar{I}}{\mathrm{z}}$ with a particular condition. They allow scientists to see which gene variants pop up more frequently in people who have a disorder.

Finding small genetic signals is a question of statistics: a weak association between a gene and a disease may stray into significance only when a study has hundreds or thousands of participants. But instead of helping to firm up which genes might be candidates, the largest population studies completed so far in psychiatric genetics seem to be eliminating them. A study this year led by Patrick Sullivan, a geneticist at the University of North Carolina at Chapel Hill, involved nearly 750 patients with schizophrenia and a similar number of controls, and analysed almost half a million SNPs. But not one gene met the rigorous statistical requirements needed to show it was a risk factor $^{3}$ - not even DISC1. 
deny that it is a crude approximation and that the symptoms used to assign categories substantially overlap. Delusions are frequently a symptom of schizophrenia, but a typical person with schizophrenia might also exhibit apathy. Patients with major depressive disorder usually exhibit apathy, but some will also be delusional. A clinical diagnosis "may be the mental end-stage of all sorts of different neurobiological disorders", says Robin Murray, a psychiatrist from Kings College London. "It may be a little like a diagnosis of 'renal failure' which is the end-stage of all sorts of medical disorders from heart disease to toxic shock."

This presents a profound problem for genetic studies because it means that some researchers may be looking for shared risk genes in a group of people who may not actually share any or, indeed, have the same disease. It is an absolutely crucial point; many researchers think that poor and inconsistent diagnoses may explain much of the past failure of genetic-association studies and will be the factor on which future success hinges. The difficulties became apparent even before the genome-association studies. The DISC1 story - in which a strong candidate identified in one study is rarely found in another - has been repeated over and over in family studies and in case-control studies, in which groups of unrelated individuals with a disorder are compared with healthy controls.

Weinberger is convinced that the right signals will be found by his candidategene approach, and says that millions of the Lieber family's money will be spent to that end. Weinberger likens the huge endowment to the Manhattan Project in its ambition and scope. "We'll try to work out the risk genes, work out the faulty circuitry and work out how to develop new ways of treatment," he says.

Weinberger's first major breakthrough involved a gene called COMT, which encodes a key enzyme in the metabolism of dopamine, a neurotransmitter that is important in schizophrenia and the target of many antipsychotic drugs.

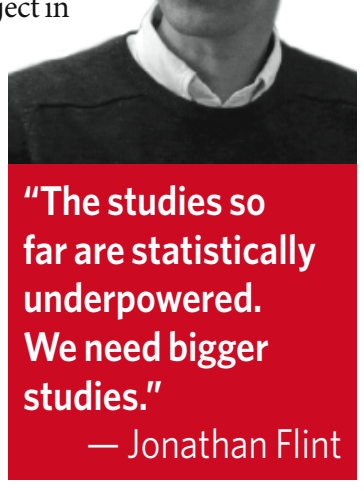

COMT that causes a methionine amino acid to be substituted for a valine in the protein tended to have worse working memory and lower activity in the prefrontal cortex than those without the mutation. They also found that the mutated version of the gene and the poorer working memory were found in more parents whose offspring had schizophrenia than in those who didn't ${ }^{4}$. That makes biological sense, says Weinberger, because the two forms of the enzyme differ in how efficiently they break down dopamine, so children who inherit the methionine version would have upset levels of a key transmitter that might make them more susceptible to schizophrenia.

The study was a turning point in the field, says Jeremy Hall, a neuroscientist at the University of Edinburgh, UK, who was one of those convinced to adopt the approach.

But Flint argues that no one has yet shown that intermediate phenotypes have a tighter link to the genes responsible than the disease itself. "I just don't buy that brain size or whatever will work better," he says.

Weinberger quickly followed COMT had already been linked to schizophrenia in family and case-control studies. But Weinberger instead examined whether variation in the gene was linked to one of his intermediate phenotypes. In this case he used functional magnetic resonance imaging (fMRI) to examine the activity of the brain's prefrontal cortex, which is known to function abnormally in schizophrenia.

In a 2001 paper, Weinberger and his colleagues showed that people with a variation in his 2001 paper with another in which he examined variants of the serotonin transporter gene that are known to be risk factors in psychiatric disorders such as depression. Rather than looking for an association between genetic variants and a specific disorder, Weinberger looked for links with another intermediate phenotype. He used fMRI to show that those with the 'short' version of the gene - who have less efficient serotonin signalling - also had more activity in the amygdala when they reacted to fearful stimuli such as angry or afraid faces ${ }^{5}$.

It was the first time that anyone had shown that a gene could change how a region of the brain responds to an emotional stimulus - the idea being that it could predispose someone to a range of psychiatric conditions if he or she encounters the right environmental trigger. In 2003, a study done in New Zealand independent of Weinberger identified one of those potential genes. The team used data from a long-term study that tracked 1,000 children through young adulthood, and showed that those with the short variant of the serotonin transporter gene were more likely to be thrown into serious depression when faced with a stressful life crisis, such as relationship angst, than were those with the long variant ${ }^{6}$. It's a classic case of gene-environment interaction, and Weinberger has gone on to identify other gene variants, such as one that could make children more likely to develop schizophrenia if they are exposed to a period without oxygen during birth.

\section{Emotional response}

The main criticism of the candidate gene approach is that it is restricted to the tiny list of genes that have established links to the disease, precisely what researchers are short of. "We are just too ignorant of the underlying neurobiology to make guesses about candidate genes," says neuroscientist Steven Hyman, provost of Harvard University in Cambridge, Massachusetts. "Candidate genes are like packing your own lunch box and then looking in the box to see what's in it." That's why Hyman and many other researchers say that, despite their past failures, bigger and better genome-wide association studies are a more promising way forward for psychiatric genetics.

Several meta-analyses are now under way in attempts to increase the statistical power and 


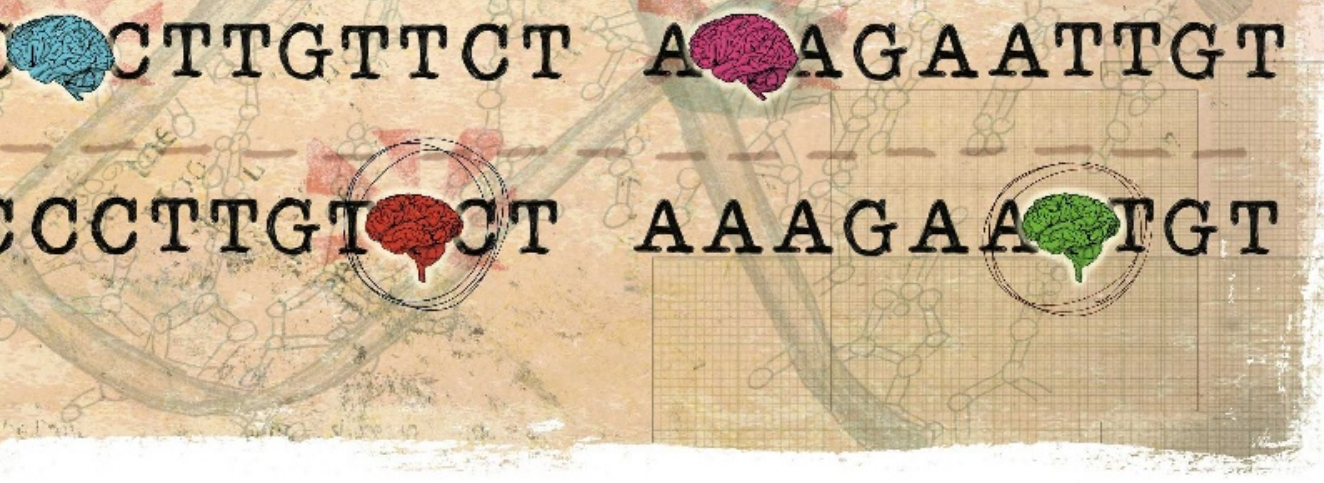

to pick up risk genes that earlier studies might have missed. One such initiative, the Psychiatric Genome-Wide Association Study Consortium, comprises groups from all over North America and Europe doing such studies. It focuses on five disorders - autism, attention-deficit hyperactivity disorder, bipolar disorder, major depressive disorder and schizophrenia, and includes some 59,000 individuals. Where possible, the scientists are pooling their data so that they can detect genetic associations that their individual efforts could not pick up alone. For example, the consortium is currently comparing 13,000 cases of major depression with 10,000 controls.

Brute numerical force might still not be enough if researchers are not exquisitely careful about the way they diagnose patients, and even Hyman expresses reservations. "There are potential differences in different patient samples the name autism or schizophrenia does not necessarily identify similar populations because of a lack of objective medical tests." If patients grouped together as having schizophrenia actually include some with other disorders, then statistical power becomes moot.

Sullivan, a member of the consortium, agrees that it will take great care to ensure that cases are comparable in terms of diagnosis and ancestry, and across different genetic technologies. He would like critics to wait for ongoing studies to be completed before pronouncing on their likely fate. "It is not the time to play Chicken Licken - 'the sky is falling, the sky is falling," he says.

One way to ensure that all recruits are handled identically is to diagnose them carefully when they are enrolled rather than relying on existing diagnoses. Flint is working with other investigators around the world to register 6,000 women with recurrent depression and 6,000 controls in such a study. The first results won't be out until 2011. "It is delayed gratification," says Ken Kendler, one of the study's principal investigators and director of the Virginia Institute of Psychiatric and Behavioural Genetics at Virginia Commonwealth University in

Richmond. "If you are in a rush, this isn't the field for you."

More immediate gratification is coming from association studies of a different type, those that look at stretches of the genome that may be duplicated or deleted from one person to the next. One study published this year found such copy number variants (CNVs) linked to schizophrenia in up to $10 \%$ of non-familial cases - those that have not been inherited ${ }^{7}$. The CNVs arose spontaneously in the affected individuals, rather than being inherited from the parents. Some of the genetic regions that are being identified have been implicated in autism, underlining how some risk genes are shared across diagnoses. These mutations tend to confer a higher risk of the disease, perhaps explaining why they are being picked up in association studies where SNPs were not, and they may turn out to be particularly useful in diagnosis.

But everything must always come back to the biology, says an unimpressed Weinberger. He likens these genome searches to a study trying to understand why road accidents happen by throwing everything that's known about drivers into the pot. "You'll find that the only thing they all have in common is ownership of a driving licence," he says. "But that's not going to give you any insight into the diverse things that really matter," such as local speed limits, foggy weather and when the local bars close. Similarly, genome-wide association studies for schizophrenia might highlight a gene linked to oxygen deprivation at birth - one of the strongest risk factors for the disorder - but that has nothing to do with the brain. "The endgame can't be statistical," Weinberger says, "it has to get down to gene variants that impact the neurobiology involved."

\section{Back to biology}

Population geneticists insist that without rigorous statistics, candidate genes, logical though they may sound, could turn out to be false leads. And once statistically validated genes are in hand, the plan is to investigate their biological role in disease, along with their interactions with other genes and the environment.

No one doubts that the animosities will defuse as more data are generated and the power of the approaches becomes clear. Whether those data will help to refine the 300 categories of disease, remains uncertain. If the same pool of risk genes underlies all the disorders, the existing groupings could vanish, only to be replaced with an even longer list in which diagnosis is based on countless genetic combinations.

Mental-health professionals are already working on the fifth edition of the diagnostic handbook. The final version is due to be released in 2012, but even that may be too soon for genetics to help substantially in reclassification. Psychiatrists are already coming to the conclusion that the DSM should be used flexibly. They realize that treatments should control the specific symptoms a patient displays - depression by antidepressants, for example, even if their formal diagnosis is schizophrenia.

With questionable diagnoses and impenetrable biology, symptoms are the best indicators that the psychiatric profession has to go on. But when the dust settles, and the data do start to flow, genetics may finally offer some clarity. Alison Abbott is Nature's senior European correspondent.

\footnotetext{
1. St Clair, D. et al. Lancet 336, 13-16 (1990).

2. Millar, J. K. et al. Hum. Mol. Genet. 9, 1415-1423 (2000)

3. Sullivan, P. F. et al. Mol. Psychiatr. 13, 570-584 (2008)

4. Egan, M. F. et al. Proc. Natl Acad. Sci. USA 98, 6917-6922 (2001).

5. Hariri, A. R. et al. Science 297, 400-403 (2002).

6. Caspi, A. et al. Science 301, 386-389 (2003).

7. Xu, B. et al. Nature Genet. 40,880-885 (2008).
}

See Editorial, page 137. 